HNO 2013 $\cdot 61: 460-461$

DOI 10.1007/s00106-013-2714-3

Online publiziert: 15. Mai 2013

c) Springer-Verlag Berlin Heidelberg 2013

\title{
S. Euteneuer
}

Hals-Nasen-Ohrenklinik, Universitätsklinikum Heidelberg

Chronische

Funktionsstörungen der Tuba Eustachii

\section{Diagnostik und Ergebnisse der Ballondilatation der Tuba Eustachii}

Das Ansinnen, eine chronisch gestörte Funktion der Tuba Eustachii zu behandeln, ist untrennbar mit der modernen Ohrchirurgie verbunden. Pau fasst in seiner Übersichtsarbeit, im Jahr 2011 in dieser Zeitschrift publiziert, treffend die kritische Rolle der Tubenfunktion für die Mittelohrchirurgie zusammen: „Auch die besten Prothesen zur Wiederherstellung des Schallleitungsapparats können wenig zur Hörverbesserung beitragen, wenn eine Tubendysfunktion vorliegt." [1]. Gleichsam bedingt die chronisch gestörte Mittelohrbelüftung bei eingeschränkter Tubenfunktion erst die Entwicklung chronisch mesotympanaler bzw. epitympanaler Otitiden.

Sudhoff et al. geben zum Einstieg in dieses Leitthemenheft eine historische Übersicht über die Diagnostik- und Therapiekonzepte der Tuba Eustachii. Im 18. Jahrhundert war die Bedeutung der gestörten Tubenfunktion für die Entwicklung von chronischen Otitiden sehr präsent. Katheterisierungen, Sondierungen und Spülungen der Tuba Eustachii wurden an größeren Patientenkollektiven durchgeführt. Damit steht die im Jahr 2009 in Bielefeld etablierte und im Jahr 2010 erstmals klinisch publizierte Methode der Ballondilatation der Tuba Eustachii $[2,3]$ in einer langen Kette von Interventionsverfahren an der Tuba Eustachii. Im 20. Jahrhundert ist, parallel zur Entwicklung des Ohrmikroskops, die Behand- lung der dysfunktionellen Tube sowie die Möglichkeit zur Mittelohrbelüftung über den Gehörgang mittels Paukendrainagen praktisch in Vergessenheit geraten. - Erst in den letzten 5 Jahren erlebt sie eine erneute Renaissance. Mit den heutigen technischen Möglichkeiten in Diagnostik und Therapie ist die Behandlung der Tubendysfunktion damit im neuen Jahrtausend angekommen.

Boeckers legt mit ihren Erläuterungen der Bestandteile des Organsystems der Tuba Eustachii den Grundstein für das Verständnis der Physiologie und Pathophysiologie der Tubenfunktion. Es wird deutlich, dass die Tuba Eustachii kein einfaches aufzudehnendes Röhrchen ist, sondern vielmehr ein komplexes funktionelles System aus Schleimhaut, Knorpel und der darin inserierenden Muskulatur. Jeder dieser Bestandteile scheint zur Funktionsstörung beizutragen, auch wenn die Interaktion im Funktionssystem und deren Manipulation durch die Ballondilatation bisher noch nicht vollständig verstanden ist.

Die Vielzahl der gebräuchlichen Funktionstests der Tuba Eustachii macht deutlich, dass es kein alleiniges Verfahren gibt, das in der Lage ist alle relevanten Aspekte der Tubenfunktion abzubilden. Di Martino bietet in seiner Übersichtsarbeit ein umfassendes Update der klinischen und apparativen Funktionstests der Tuba Eustachii. Seit den frühen Interventionen an 
der Tuba Eustachii im 18. Jahrhundert begleiten Bedenken hinsichtlich der fatalen Verletzung der in direkter Nachbarschaft verlaufenden $A$. carotis interna die Interventionsverfahren. So wird bisher von den meisten Anwendern der Ballondilatation präoperativ die CT-morphologische Abklärung des Karotiskanals durchgeführt. Tisch et al. präsentieren eine in dieser Hinsicht bedeutende Arbeit: Im Routinekollektiv der klinischen HNO-Praxis konnten keine knöchernen Dehiszenzen des Karotiskanals identifiziert werden. Die präoperative CT wird damit in $\mathrm{Zu}$ kunft wohl sparsamer und gezielter eingesetzt werden können.

Die Einjahresdaten von Sudhoff et al., zusammengefasst im Abschluss seiner Übersicht, sowie die Daten von Tisch et al. belegen die Sicherheit und mindestens mittelfristige Effektivität des Verfahrens bei Erwachsenen. Vor dem Hintergrund dieser bisherigen Erfolge ist es nur konsequent, dass Tisch et al. mit einem Beitrag über eine erste Anwendung beim Kind provozieren.

Die Fachgesellschaft darf auf die Langzeitergebnisse aus den Kliniken, in denen seit längerem dilatiert wird, gespannt sein. Gemäß den bisherigen Ergebnissen ist die Ballondilatation als eine viel versprechende, praktikable Therapiealternative zur klassischen Paukendrainage in der Therapie der chronischen Funktionsstörung der Tuba Eustachii zu empfehlen.

\section{Literatur}

1. Pau HW (2011) Tube und Mittelohrmechanik. HNO 59: 953-963

2. Ockermann T, Reinecke U, Upile T, Ebmeyer J, Sudhoff $\mathrm{H}$ (2010) Ballondilatation eustachian tuboplasty: a clinical study. Laryngoscope 120: 14111416

3. Schroeder S, Reineke U, Lehmann M, Ebmeyer J, Sudhoff H (2013) Chronisch obstruktive Tubenfunktionsstörung des Erwachsenen. HNO 61: 142151

Dr. med. S. Euteneuer

\section{Korrespondenzadresse}

Dr. S. Euteneuer
Hals-Nasen-Ohrenklinik, Uni-
versitätsklinikum Heidelberg
Im Neuenheimer Feld 400,
69120 Heidelberg
sara.euteneuer@med.uni-hei-
delberg.de

Interessenkonflikt. Die korrespondierende Autorin gibt an, dass kein Interessenkonflikt besteht. 